Methods: This is a cross-sectional study that consecutively included 14 patients (100\% female, mean age 55.3 years) with RA (median duration of evolution of 13.5 years, mean specialised care of 2.1 years). The functional status of RA was assessed by the HAQ (Health Assessment Questionnaire). RA activity was evaluated by DAS28 and DAS44. The FFI score (Foot Function Index) was used to determine the functional status of the foot. A correlation analysis was made between the FFI score and DAS28, DAS44 and HAQ as well as with the duration of progression of the RA and the delay of specialised management.

Results: The median score of the HAQ (Health Assessment Questionnaire) was 1.6. The median score for the Foot Function Index (FFI) was 53.6. The medians of DAS 28 and DAS 44 were 5.3 and 3.8. The median of the different FFI score items was:

Pain Sub-scale: How severe is your foot pain?

Q1: Foot pain at its worst ? $7(6 ; 9,25)$, Q2: Foot pain in morning ? 5,5 (2; 7), Q3: Pain walking barefoot ? $4(1,75 ; 8,15)$, Q4: Pain standing barefoot ? $5(3,25 ; 8,25)$, Q5: Pain walking with shoes ? $4(1,75 ; 5,75)$, Q6: Pain standing with shoes ? 4,5 $(3 ; 5,5)$, Q7: Pain walking with orthotics ? $3(3 ; 3)$, Q8: Pain standing with orthotics ? $3(3 ; 3)$, Q9: Foot pain at end of day ? $5(2,75 ; 7)$

Disability sub-scale: How much difficulty did you have?

Q10: Difficulty walking in house ? $2(0 ; 3,25)$, Q11: Difficulty walking outside ? 5,5 (3; 8), Q12: Difficulty walking 4 blocks ? 5 (4; 9), Q13: Difficulty climbing stairs ? $6,5(4 ; 8,25)$, Q14: Difficulty descending stairs ? $6(3,5 ; 8,25)$, Q15: Difficulty standing tip toe ? 8,5 (5,25; 10), Q16: Difficulty getting up from chair ? $5(1,5 ; 8)$, Q17: Difficulty climbing curbs ? 4,5 (2; 7,25), Q18: Difficulty walking fast ? 8,5 $(4,75 ; 10)$ Activity Limitation sub-scale: How much of the time do you?

Q19: Stay inside all day because of feet ? $7(2 ; 8,25)$, Q20: Stay in bed all day because of feet ? $5(2 ; 3,25)$, Q21: Limit activities because of feet ? 5,5 $(1 ; 8)$, Q22: Use assistive device (cane, walker, crutches, etc) indoors ? $0(0 ; 3,25)$, Q23: Use assistiw device (cane, walker, crutches, etc) outdoors ? $0(0 ; 0,75)$.

No correlation was found between the FFI and the HAQ. DAS 28 and DAS44 did not correlate with the $\mathrm{FFI}$ either.

Alteration of foot function as indicated by a high FFI score was associated with a delay in specialist management by a rheumatologist $(r=0.535, p=0.04)$.

Conclusions: This study provides insight into the impact of RA on foot function. The HAQ, DAS28 and DAS44 would not reflect the functional deterioration of the foot in RA. In addition, an alteration of foot function would be associated with a delay in specialist management by a Rheumatologist.

A large-scale study is underway to validate and complete these preliminary results.

Disclosure of Interest: None declared

DOI: 10.1136/annrheumdis-2018-eular.6774

\section{AB0305 REMAINING FOOT SYNOVITIS MAY PREDICT RELAPSE IN RHEUMATOID ARTHRITIS PATIENTS IN DAS28 REMISSION (DAS28-CRP<2.3)}

S. Hattori ${ }^{1}$, N. Hagino ${ }^{1}$, K. Yomono ${ }^{1}$, S. Tsuzuki ${ }^{1}$, S. Suzuki ${ }^{1}$, S. Takenouchi ${ }^{1}$, R. Yokochi ${ }^{1}$, T. Matsui ${ }^{2}$, S. Tohma ${ }^{3}$. 'Division of Hematology and Rheumatology, Teikyo University Chiba Medical Center, Ichihara; ${ }^{2}$ Department of Rheumatology, Clinical Research Center for Allergy and Rheumatology, Sagamihara National Hospital, Sagamihara; ${ }^{3}$ Department of Rheumatology, Tokyo National Hospital, Kiyose, Japan

Background: DAS28 (Disease Activity Score based on 28 joints) is widely used to determine remission in rheumatoid arthritis (RA). The problems with DAS28 due to omission of the lower extremity joints have long been discussed. There are reports demonstrating that some patients meeting DAS28 remission criteria still have synovitis in their feet. ${ }^{1}$ It is suggested that patients with remaining foot synovitis can experience up to a 2 -fold increase in relapse rates. ${ }^{2}$ Evidence supporting this hypothesis is still scarce.

Objectives: We hypothesised that RA patients in remission according to the DAS28 remission criterion with remaining foot synovitis are less likely to stay in remission. We aimed to test this hypothesis among Japanese patients with $R A$. Methods: In 2015, 15,071 RA patients were registered in a large nationwide database in Japan (NinJa). Among those patients, those who were in remission based on DAS28-CRP were included. We used DAS28-CRP $<2.3$ as the remission criteria. The study population was then divided into groups with or without tender/swollen joints in the feet. These patients were followed for one year and the remission rate was then calculated for each group. Comparison was performed with Chisquare test.

Results: We included 706 RA patients in remission based on DAS28-CRP with foot synovitis in 2015 and 5182 without foot synovitis. Among patients with foot synovitis in 2015, 225 (31.9\%) were not in remission in 2016 while among those without foot synovitis, $985(19.0 \%)$ were not in remission. (X-squared $=62.953$, $\mathrm{df}=1, \mathrm{p}=2.117 \times 10-1^{5}$ )

Conclusions: In our study, RA patients in remission according to the DAS28 remission criterion with remaining foot synovitis had more relapses than those without it. Remission defined by DAS28-CRP does not take remaining foot synovitis into account. But a thorough foot examination may still be useful in RA patients with DAS28-CRP remission as they may be more at risk for relapse.

\section{REFERENCES:}

[1] van der Leeden, et al. Arthritis Res Ther. 2010;12,1:R3.

[2] Wechalekar MD, et al. Arthritis Care Res. 2016;68,11:1616-1623.

Disclosure of Interest: None declared

DOI: 10.1136/annrheumdis-2018-eular.3274

\section{AB0306 PREDICTORS OF MEDICATION ADHERENCE IN SERBIAN PATIENTS WITH RHEUMATOID ARTHRITIS}

S. Zivojinovic ${ }^{1}$, J. Nedovic ${ }^{2}$, T. Jankovic ${ }^{3}$, N. Damjanov ${ }^{4}$, on behalf of Prof Nemanja Damjanov. ${ }^{1}$ Clinical V, Institute of Rheumatology, Belgrade; ${ }^{2}$ Institute For Treatment and Rehabilitation Niska Banja, Nis; ${ }^{3}$ Clinic For Rheumatic disease, Novi Sad; ${ }^{4} \mathrm{Head}$ of the Institute of Rheumatology, Institute of Rheumatology, Belgrade, Serbia

Background: Adherence to pharmacologic therapy is a significant problem in patients with rheumatoid arthritis (RA). Nonadherence in patients with RA using disease modifying antirheumatic drugs (DMARD) may result in unnecessarily high levels of disease activity and function loss

Objectives: The aim of this study was to evaluate the predictive ability of demographic (age, sex, marital status, employment status, education) and clinical factors (duration of disease, patients assessment of pain, concomitant use of biological therapy and presence of comorbidity) for medication adherence.

Methods: In the period between March 1 and May 30, 2017, 195 consecutive RA patients who were treated in one of three randomly chosen Serbian clinics were enrolled in cross-sectional study. The inclusion criteria were: age $\geq 18$ years, current diagnosis of RA per the 1987. ACR Diagnostic Criteria, completing the ques tionnaire self-reported medication adherence and written informed consent Demographic and clinical characteristics of adherent and non-adherent participants were compared using independent samples t-tests for continuous variables and Chi square analyses for categorial variables. The associations between investigated demographic and clinical characteristics of the patients and nonadherence to DMARDs were assessed applying logistic regression analysis.

Results: Study population was predominantly female $(88 \%)$, and the average current age was $57.4 \pm 11.2$ years. The median duration of RA was 9 years. Only one quarter $(25 \%)$ of participants were employed, those unemployed $26 \%$ or retired $(48 \%)$ comprised the majority of the sample. In terms of education approximately $60 \%$ participants finished secondary school. The participants in this study were primarily married $(77 \%)$. The majority of the patients were prescribed methotrexate $(77 \%)$, other common DMARDs prescribed for these patients included hydroxychloroquine (13\%) and prednisone (18\%). Approximately $20 \%$ of patients were receiving a biological drug. Half of the patients estimated that they had severe pain on visual analogue scale (VAS $>5$ ). The majority of the RA patients had some comorbidity ( $80 \%)$. The most of these RA patients $(89.7 \%)$ were considered adherent to medication prescriptions and the remainder $(11.3 \%)$ were nonadherent. There were no significant difference in clinical or demographic factors between groups except for employment and concomitant use of biological therapy. One third (33.3\%) of the non-adherent participants were using biologic drug while only $19 \%$ of the adherent patients were using biologics. There were significantly more employees (29\%) among the non-adherent patients. According to the results of univariate regression analysis the following factors are significantly associated with non-adherence to DMARDs: employment $(O R=2.277 ; p=0.049)$, and concomitant use of biological therapy $(O R=2.312 ; p=0.002)$. Finally, in the multivariate regression model concomitant use of biological therapy $(\mathrm{OR}=2.067$ $\mathrm{p}=0.017$ ) remained statistically significant and was identified as independent predictor of non-adherence to DMARDs.

Conclusions: The results of our study have shown that about $10 \%$ of RA patients met the criteria for non-adherence to DMARDs. Concomitant use biologics and employment are independent predictors of non-adherent.

Disclosure of Interest: None declared

DOI: 10.1136/annrheumdis-2018-eular.4273 